\title{
Photon correlations between the lines in the spectrum of resonance fluorescence
}

\author{
$\mathrm{H} F$ Arnoldus and $\mathrm{G}$ Nienhuis \\ Fysisch Laboratorium, Rijksuniversiteit Utrecht, Postbus 80000 , 3508 TA Utrecht, The \\ Netherlands
}

Received 26 July 1983, in final form 31 October 1983

\begin{abstract}
We give a theoretical description of the spectrally resolved intensity correlations of an electromagnetic field, in analogy with the definition of the physical time-dependent spectrum. The results are applied to evaluate the time correlations between the three lines in the spectrum of resonance fluorescence of a two-level atom. We include the possibility of a finite bandwidth of the incident radiation, and we allow for collisions with perturber atoms. Photons in the central Rayleigh line are emitted in a fully random fashion, without any correlation with previous or subsequent emissions. Two photons from the same sideband display antibunching in time, whereas two photons from opposite sidebands tend to bunch with a strong asymmetry in time. The effect of collisions and of a finite bandwidth is to diminish this asymmetry.
\end{abstract}

\section{Introduction}

Intensity correlations in the fluorescent emission of atoms display the celebrated phenomenon of antibunching in time (Carmichael and Walls 1976, Kimble and Mandel 1976, Wódkiewicz 1980). This feature is particularly interesting, since it represents an essentially quantum-mechanical property of the fluorescence field, which cannot be reproduced by any classical field (Paul 1982).

It is well known that for large detunings from resonance, or for large Rabi frequencies, the spectrum of resonance fluorescence of a two-level atom contains three separate lines, i.e. the central Rayleigh component at the incident frequency, and two sidebands, which are usually termed the three-photon line and the fluorescence line (Mollow 1969, Carlsten et al 1977). The question arises in which way the overall intensity correlation of the integrated emission is distributed over the various combinations of lines. This question has been discussed by Apanasevich and Kilin (1979) and by Cohen-Tannoudji and Reynaud (1979). These authors consider the case of a monochromatic driving field incident on a free two-level atom. It was found that photon emissions in the central line of the three-line spectrum are not correlated to previous or later emissions. Photons from a single sideband display antibunching, whereas emissions from opposite sidebands are bunched with a considerable asymmetry in their time ordering. These results are in line with recent experimental observations by Aspect et al (1980), who found that emissions in the three-photon line tend to precede emissions in the fluorescence line.

In the present paper we give a general formal expression for the two-time intensity correlation function with spectral resolution. This expression takes the form of a 
double convolution of a quasi-distribution, which is determined by field properties only, and a smoothing function that contains the properties of the frequency filters. We apply this result to the derivation of the spectrally resolved intensity correlation function of resonance fluorescence. We allow for collisions with a perturber gas, and for phase fluctuations of the incident field, which result in a finite bandwidth. In the special case of the three-line fluorescence spectrum of a two-level atom we find that increasing the incident intensity can either increase or decrease the correlation time between successive emissions, depending on whether the bandwidth and the collisional width are larger or smaller than the natural width. Finally, collisions and phase fluctuations decrease the asymmetry in the time ordering of photons from opposite sidebands.

\section{Time correlation between frequency-resolved photons}

The number of detected photons per unit time by a photomultiplier is proportional to the local energy density of the electromagnetic field $2 \varepsilon_{0}\left\langle\boldsymbol{E}^{(-)}(r, t) \cdot \boldsymbol{E}^{(+)}(r, t)\right\rangle$, with $\boldsymbol{E}^{(+)}$the positive-frequency (annihilation) part of the electric-field component. If we wish to count only photons within a small frequency range around a central frequency $\omega_{0}$, we have to filter the field by passing it through a spectrometer with $\omega_{0}$ as its setting frequency. The annihilation part of the resulting filtered field is related to the original field by

$$
\overline{\boldsymbol{E}}^{(+)}(t)=\int_{0}^{\infty} \mathrm{d} \tau \exp \left(-\mathrm{i} \omega_{0} \tau\right) f(\tau) \boldsymbol{E}^{(+)}(t-\tau)
$$

(Renaud et al 1977, Eberly and Wódkiewicz 1977) with $f(t)$ a filter function, which we shall suppose to be real. The component of $\boldsymbol{E}^{(+)}$in the direction of the detected polarisation is denoted as $E^{(+)}$, and we suppress the $r$ dependence. The number of detected photons from the filtered field per unit time is now represented as

$$
\bar{I}_{1}\left(t, \omega_{0}\right)=\gamma\left\langle\bar{E}^{(-)}(t) \bar{E}^{(+)}(t)\right\rangle
$$

with $\gamma$ a proportionality constant. This expression (2.2) is termed the physical spectrum by Eberly and Wódkiewicz (1977). It has been demonstrated (Nienhuis 1983) that $\bar{I}_{1}\left(t, \omega_{0}\right)$ is directly related to the definition of Page (1952) and Lampard (1954) of a time-dependent spectrum

$$
I_{1}(t, \omega)=(\gamma / \pi) \operatorname{Re} \int_{0}^{\infty} \mathrm{d} \tau \exp (\mathrm{i} \omega \tau)\left\langle E^{(-)}(t-\tau) E^{(+)}(t)\right\rangle .
$$

The physical spectrum $\bar{I}_{1}$ is a convolution over time and frequency of the quasi-spectrum (2.3) with a smoothing function

$$
s(\omega, \tau)=2 \operatorname{Re} \int_{0}^{\infty} \mathrm{d} \tau^{\prime} \exp \left(-\mathrm{i} \omega \tau^{\prime}\right) f(\tau) f\left(\tau+\tau^{\prime}\right)
$$

according to the simple relation

$$
\bar{I}_{1}\left(t, \omega_{0}\right)=\int \mathrm{d} \omega \int_{0}^{\infty} \mathrm{d} \tau s(\omega, \tau) I_{1}\left(t-\tau, \omega_{0}-\omega\right) .
$$

The smoothing function (2.4) is completely determined by the properties of the 
spectrometer, whereas the quasi-spectrum (2.3) depends only on the field. The function $s(\omega, \tau)$ has a frequency width and a time width which are related by the uncertainty relation.

In the present paper we discuss the two-time probability density of spectrally resolved photons detected from a radiation field. From the first part of this section it will be obvious that spectral resolution of the detected photons is accounted for if we simply replace the incident field $E^{(+)}(t)$ by the filtered field $\bar{E}^{(+)}(t)$. On the other hand, for a measurement of a photon with a frequency around $\omega_{1}$ at time $t_{1}$, and a photon with a frequency around $\omega_{2}$ at time $t_{2}$, one has to use two spectrometers and two multipliers. The first spectrometer modifies the incident field $E^{(+)}(t)$ into the filtered field $\bar{E}_{1}^{(+)}(t)$, and the second one gives the filtered field $\bar{E}_{2}^{(+)}(t)$. These two filtered fields are given by equation (2.1) when the setting frequency $\omega_{0}$ is replaced by the frequencies $\omega_{1}$ and $\omega_{2}$, respectively. For convenience we assume that the two field functions $f$ pertaining to the two spectrometers are equal, but this is by no means essential. The two-time probability density for counting a photon at time $t_{1}$ with a frequency within the passband width around the setting frequency $\omega_{1}$, and a second photon at time $t_{2}$ with a frequency around $\omega_{2}$ is then given by the intensity correlation function (Glauber 1965, Kelley and Kleiner 1964)

$$
\bar{I}_{2}\left(t_{1}, \omega_{1} ; t_{2}, \omega_{2}\right)=\gamma^{2}\left\langle\bar{E}_{1}^{(-)}\left(t_{1}\right) \bar{E}_{2}^{(-)}\left(t_{2}\right) \bar{E}_{2}^{(+)}\left(t_{2}\right) \bar{E}_{1}^{(+)}\left(t_{1}\right)\right\rangle
$$

for $t_{2} \geqslant t_{1}$. One notices that $\bar{I}_{2}$ is a non-negative function of two times and two frequencies, as it should be. A two-photon counting experiment with spectral resolution was recently performed for resonance fluorescence by Aspect et al (1980).

We substitute equation (2.1) for different times $t=t_{1}, t_{2}$ and different setting frequencies $\omega_{0}=\omega_{1}, \omega_{2}$ into (2.6), and we use the inverse of equation (2.4) for $\tau^{\prime} \geqslant 0$

$$
f(\tau) f\left(\tau+\tau^{\prime}\right)=\frac{1}{2 \pi} \int \mathrm{d} \omega \exp \left(\mathrm{i} \omega \tau^{\prime}\right) s(\omega, \tau)
$$

We find that $\bar{I}_{2}$ can be expressed as a double convolution of a quasi-distribution function $I_{2}$, in analogy with $(2.5)$

$$
\begin{aligned}
\bar{I}_{2}\left(t_{1}, \omega_{1} ; t_{2}, \omega_{2}\right) \\
=\int \mathrm{d} \omega_{1}^{\prime} \int_{0}^{\infty} \mathrm{d} \tau_{1} \int \mathrm{d} \omega_{2}^{\prime} \int_{0}^{\infty} \mathrm{d} \tau_{2} s\left(\omega_{1}^{\prime}, \tau_{1}\right) s\left(\omega_{2}^{\prime}, \tau_{2}\right) \\
\quad \times I_{2}\left(t_{1}-\tau_{1}, \omega_{1}-\omega_{1}^{\prime} ; t_{2}-\tau_{2}, \omega_{2}-\omega_{2}^{\prime}\right)
\end{aligned}
$$

where the quasi-distribution function

$$
\begin{aligned}
& I_{2}\left(t_{1}, \omega_{1} ; t_{2}, \omega_{2}\right) \\
& =\frac{\gamma^{2}}{2 \pi^{2}} \operatorname{Re} \int_{0}^{\infty} \mathrm{d} \tau_{1}^{\prime} \int_{0}^{\infty} \mathrm{d} \tau_{2}^{\prime}\left[\exp \left(\mathrm{i} \omega_{1} \tau_{1}^{\prime}+\mathrm{i} \omega_{2} \tau_{2}^{\prime}\right)\right. \\
& \times\left\langle E^{(-)}\left(t_{1}-\tau_{1}^{\prime}\right) E^{(-)}\left(t_{2}-\tau_{2}^{\prime}\right) E^{(+)}\left(t_{2}\right) E^{(+)}\left(t_{1}\right)\right\rangle \\
& \left.+\exp \left(\mathrm{i} \omega_{1} \tau_{1}^{\prime}-\mathrm{i} \omega_{2} \tau_{2}^{\prime}\right)\left\langle E^{(-)}\left(t_{1}-\tau_{1}^{\prime}\right) E^{(-)}\left(t_{2}\right) E^{(+)}\left(t_{2}-\tau_{2}^{\prime}\right) E^{(+)}\left(t_{1}\right)\right\rangle\right]
\end{aligned}
$$

depends only on the incident field. A large passband width of the spectrometer, corresponding to a large spectral width of the smoothing functions, gives rise to a small range of values of $\tau_{1}$ and $\tau_{2}$ contributing to (2.8), and likewise the integration over $\omega_{1}^{\prime}$ and $\omega_{2}^{\prime}$ in (2.8) restricts the range of values of $\tau_{1}^{\prime}$ and $\tau_{2}^{\prime}$ contributing to $\bar{I}_{2}$. 


\section{Fluorescence radiation}

In order to fix the notation, we now give a brief summary of the theory of fluorescence radiation from a two-level atom in a non-monochromatic radiation field. We shall demonstrate that the evaluation of the two-time quasi-distribution function $I_{2}$ can be performed along the same lines that lead to the fluorescence spectrum.

We include a finite bandwidth $\lambda_{1}$ of the incident radiation as resulting from stochastic fluctuations in the phase $\psi(t)$ of the electric field

$$
\boldsymbol{E}(t)=E_{0} \operatorname{Re} \varepsilon_{\mathrm{L}} \exp \left[-\mathrm{i}\left(\omega_{\mathrm{L}} t+\psi(t)\right)\right] .
$$

The atomic dipole $\boldsymbol{\mu}$ interacts with the external field, giving rise to a coupling strength $\hbar \Omega=E_{0}\left\langle e\left|\mu \cdot \varepsilon_{L}\right| g\right\rangle$, where $|e\rangle$ and $|g\rangle$ are the atomic excited state and ground state. In the rotating-wave approximation the interaction is given by

$$
H_{\mathrm{ar}}(t)=-\frac{1}{2} \hbar \Omega d \exp \left[-\mathrm{i}\left(\omega_{\mathrm{L}} t+\psi(t)\right)\right]+\text { Hermitian conjugate }
$$

where $d=|e\rangle\langle g|$ is the raising part of the dipole operator. Atomic decay due to spontaneous emission is taken into account in terms of the effective relaxation operator $\Gamma$, acting on a density matrix according to the equality

$$
\Gamma \rho=\frac{1}{2} A\left(P_{\mathrm{e}} \rho+\rho P_{\mathrm{e}}-2 d^{\dagger} \rho d\right)
$$

where $A$ is the Einstein coefficient and $P_{\mathrm{e}}=d d^{\dagger}=|e\rangle\langle e|$ is the projector on the excited state. The energy separation between the atomic states is $\hbar \omega_{0}$, and the evolution of the density matrix is determined by the stochastic differential equation

$$
\mathrm{i} \hbar \frac{\mathrm{d}}{\mathrm{d} t} \rho(t)=\left[\hbar \omega_{0} P_{\mathrm{e}}+H_{\mathrm{ar}}(t), \rho(t)\right]-\mathrm{i} \hbar \Gamma \rho(t) .
$$

The rapid oscillation of the Hamiltonian with the optical frequency $\omega_{\mathrm{L}}$ of the driving field can be transformed away by applying the unitary transformation

$$
\sigma(t)=\exp \left[\mathrm{i}\left(\omega_{\mathrm{L}} t+\psi(t)\right) K\right] \rho(t)
$$

with

$$
K \rho=\left[P_{\mathrm{e}}, \rho\right] .
$$

Then $\sigma$ obeys the Liouville equation

$$
\mathrm{i} \frac{\mathrm{d}}{\mathrm{d} t} \sigma(t)=\left(L_{\mathrm{d}}-\mathrm{i} \Gamma-\dot{\psi}(t) K\right) \sigma(t)
$$

with $\hbar L_{\mathrm{d}}$ the commutator with the dressed-atom Hamiltonian

$$
H_{\mathrm{d}}=-\frac{1}{2} \hbar\left[\Delta\left(P_{\mathrm{e}}-P_{\mathrm{g}}\right)+\Omega\left(d+d^{\dagger}\right)\right]
$$

in terms of the Rabi frequency $\Omega$ and the detuning from resonance $\Delta=\omega_{L}-\omega_{0}$. If we treat $\psi(t)$ as a process with random independent increments, the stochastic average of (3.7) has the solution (Arnoldus and Nienhuis 1983 and references therein)

$$
\langle\langle\sigma(t)\rangle\rangle=Z_{0}\left(t-t_{0}\right)\left\langle\left\langle\sigma\left(t_{0}\right)\right\rangle\right\rangle=\exp \left[-\mathrm{i}\left(t-t_{0}\right) L_{0}\right]\left\langle\left\langle\sigma\left(t_{0}\right)\right\rangle\right\rangle
$$

for $t \geqslant t_{0}$, with

$$
L_{0}=L_{\mathrm{d}}-\mathrm{i} \Gamma-\mathrm{i} W_{0}
$$


and $W_{0}$ an effective relaxation operator accounting for the finite bandwidth, given by

$$
W_{0} \sigma=\lambda_{1}\left(P_{\mathrm{g}} \sigma P_{\mathrm{e}}+P_{\mathrm{e}} \sigma P_{\mathrm{g}}\right)
$$

The stationary state $\bar{\sigma}$ of the atom is determined by the eigenvalue equation

$$
L_{0} \bar{\sigma}=0 \text {. }
$$

The spectral distribution of the fluorescence radiation is determined by the Fourier transform of the dipole correlation function. This follows from the fact that the electric-field component of the scattered radiation is proportional to the atomic lowering operator in the Heisenberg picture (Kimble and Mandel 1976)

$$
E^{(+)}(t) \propto d^{\dagger}(t)
$$

and therefore field correlation functions are proportional to the dipole correlation functions

$$
C_{n}\left(t_{1}^{\prime}, \ldots, t_{n}^{\prime} ; t_{n}, \ldots, t_{1}\right)=\left\langle d\left(t_{1}^{\prime}\right) \ldots d\left(t_{n}^{\prime}\right) d^{\dagger}\left(t_{n}\right) \ldots d^{\dagger}\left(t_{1}\right)\right\rangle
$$

The quasi-spectrum (2.3) of the fluorescence is now

$$
I_{1}(t, \omega)=\frac{A}{\pi} \operatorname{Re} \int_{0}^{\infty} \mathrm{d} \tau \exp (\mathrm{i} \omega \tau) C_{1}(t-\tau ; t)
$$

which is still a stochastic function of time. The explicit time dependence disappears after stochastic averaging. The evaluation of $C_{1}$ is easily carried out after applying the transformation (3.5) (Arnoldus and Nienhuis 1983), with the result for $\tau \geqslant 0$

$C_{1}(t-\tau, t)=\exp \left[-\mathrm{i}\left(\omega_{\mathrm{L}} \tau+\psi(t)-\psi(t-\tau)\right)\right] \operatorname{Tr} d^{\dagger} Y(t, t-\tau)(\sigma(t-\tau) d)$

where $Y\left(t, t_{0}\right)$ is the propagator of $\sigma$ according to the relation

$$
\sigma(t)=Y\left(t, t_{0}\right) \sigma\left(t_{0}\right)
$$

Hence $Y$ obeys the same Liouville equation (3.7) as $\sigma$. As shown before, stochastic averaging of (3.16) gives the result (Arnoldus and Nienhuis 1983)

$$
\left\langle\left\langle C_{1}(t-\tau, t)\right\rangle\right\rangle=\exp \left(-\mathrm{i} \omega_{\mathrm{L}} \tau\right) \operatorname{Tr} d^{\dagger} Z_{+}(\tau)(\bar{\sigma} d) .
$$

Here $Z_{+}(t)$ follows from $Z_{0}(t)$ as defined in (3.9) by replacing $W_{0}$ by $W_{+}$, defined by

$$
W_{+} \sigma=\lambda_{1}\left(P_{\mathrm{e}} \sigma P_{\mathrm{e}}+P_{\mathrm{g}} \sigma P_{\mathrm{g}}\right)+\lambda_{2} P_{\mathrm{g}} \sigma P_{\mathrm{e}}
$$

where $\lambda_{1}$ is the decay rate of the field correlation function of the incident radiation, which is equal to the bandwidth, and $\lambda_{2}$ is the decay rate of the correlation function of the square of the electric field. It is known (Swain 1980) that Gaussian phase fluctuations can be accounted for by simple substitution rules. The fluorescence spectrum in the steady state is now

$$
\left\langle\left\langle I_{1}(t, \omega\rangle\right\rangle=\frac{A}{\pi} \operatorname{Re} \operatorname{Tr} d^{\dagger} \mathrm{i}\left(\omega-\omega_{\mathrm{L}}-L_{+}\right)^{-1}(\bar{\sigma} d)\right.
$$

with

$$
L_{+}=L_{\mathrm{d}}-\mathrm{i} \Gamma-\mathrm{i} W_{+} .
$$

As a result of the stochastic averaging, this spectrum is independent of time.

The effect of collisions of the atom with foreign-gas particles can be incorporated in the description. The main modification is that we must add a collisional relaxation 
operator $-\mathrm{i} \Phi(0)$ to the Liouville operator $L_{0}$, equation (3.10), which affects the steady-state density matrix $\bar{\sigma}$, and we must add a frequency-dependent collision operator $-\mathrm{i} \Phi\left(\omega-\omega_{\mathrm{L}}\right)$ to $L_{+}$, equation (3.21), thereby modifying the spectrum. Explicit expressions for $\Phi$ are known in the literature (Nienhuis 1982, Arnoldus and Nienhuis 1983).

The spectrum of fluorescence separates into three lines in the limit that the precession frequency

$$
\Omega^{\prime}=\Delta\left[1+\Omega^{2} / \Delta^{2}\right]^{1 / 2}
$$

is large compared with the spontaneous decay rate, the collisional relaxation rate and the bandwidth $\lambda_{1}$ (Mollow 1969).

\section{The quasi-distribution function}

The spectrally resolved intensity correlation function $\bar{I}_{2}$ as given by (2.9) contains the quasi-distribution function $I_{2}$, equation (2.10). In general many different orderings of the time values occurring in (2.9) and (2.10) can arise, since $t_{2}-\tau_{2}$ can be smaller than $t_{1}$, or even smaller than $t_{1}-\tau_{1}$, even though $t_{2}$ is larger than $t_{1}$ in (2.9). Substituting (2.10) into (2.9) requires also substituting the values $t_{1}-\tau_{1}$ and $t_{2}-\tau_{2}$ for $t_{1}$ and $t_{2}$ in (2.10), and the resulting time arguments, $t_{1}-\tau_{1}, t_{2}-\tau_{2}, t_{1}-\tau_{1}-\tau_{1}^{\prime}, t_{2}-\tau_{2}-\tau_{2}^{\prime}$ can be ordered in six different ways. This situation simplifies considerably if we restrict ourselves to the reasonable case that the time resolution $\Delta \tau$ of the photon detection is much better than the time difference $t_{2}-t_{1}$. Hence we assume

$$
\Delta \tau \ll t_{2}-t_{1} \text {. }
$$

One notices that in the opposite case $\Delta \tau \geqslant t_{2}-t_{1}$ even the time order of the two detected photons would be unobservable. The assumption (4.1) restricts the range of interest of the four integration variables $\tau_{1}, \tau_{2}, \tau_{1}^{\prime}$ and $\tau_{2}^{\prime}$. Only small values of $\tau_{2}$ contribute to (2.9) as a result of (4.1), and we may assume that $t_{2}-\tau_{2}$ is larger than $t_{1}$. After substituting (2.10) into (2.9), and performing the integration over $\omega_{2}^{\prime}$, the range of interest of $\tau_{2}^{\prime}$ is likewise restricted to values small compared with $t_{2}-t_{1}$, since the spectral resolution $\Delta \omega$, or the spectral width of $s(\omega, \tau)$, must be larger than $\left(t_{2}-t_{1}\right)^{-1}$. Hence when the inequality (4.1) is obeyed, we only have to consider one single ordering of the time arguments

$$
t_{2}-\tau_{2} \geqslant t_{2}-\tau_{2}-\tau_{2}^{\prime} \geqslant t_{1}-\tau_{1} \geqslant t_{1}-\tau_{1}-\tau_{1}^{\prime}
$$

When we express $I_{2}$ in terms of the dipole correlation function $C_{2}$ as defined in (3.14) we obtain the equality

$$
\begin{aligned}
& I_{2}\left(t_{1}, \omega_{1} ; t_{2}, \omega_{2}\right) \\
& =\frac{A^{2}}{2 \pi^{2}} \operatorname{Re} \int_{0}^{\infty} \mathrm{d} \tau_{1}^{\prime} \int_{0}^{\infty} \mathrm{d} \tau_{2}^{\prime}\left[\exp \left(\mathrm{i} \omega_{1} \tau_{1}^{\prime}+\mathrm{i} \omega_{2} \tau_{2}^{\prime}\right) C_{2}\left(t_{1}-\tau_{1}^{\prime}, t_{2}-\tau_{2}^{\prime} ; t_{2}, t_{1}\right)\right. \\
& \\
& \left.+\exp \left(\mathrm{i} \omega_{1} \tau_{1}^{\prime}-\mathrm{i} \omega_{2} \tau_{2}^{\prime}\right) C_{2}\left(t_{1}-\tau_{1}^{\prime}, t_{2} ; t_{2}-\tau_{2}^{\prime}, t_{1}\right)\right] .
\end{aligned}
$$

As a result of the argument given above, we only have to account for values of $\tau_{2}^{\prime}$ that are smaller than $t_{2}-t_{1}$. An explicit evaluation of the stochastic average of the two dipole correlation functions occurring in (4.3) can be performed along the same lines that led to $(3.18)$, with the result

$$
\begin{aligned}
\left\langle\left\langleC _ { 2 } \left( t_{1}-\tau_{1}^{\prime},\right.\right.\right. & \left.\left.\left.t_{2}-\tau_{2}^{\prime} ; t_{2}, t_{1}\right)\right\rangle\right\rangle \\
& =\exp \left[-\mathrm{i} \omega_{\mathrm{L}}\left(\tau_{1}^{\prime}+\tau_{2}^{\prime}\right)\right] \operatorname{Tr} d^{\dagger} Z_{+}\left(\tau_{2}^{\prime}\right)\left(\left\{Z_{0}\left(t_{2}-\tau_{2}^{\prime}-t_{1}\right)\left[d Z_{+}\left(\tau_{1}^{\prime}\right)(\bar{\sigma} d)\right]\right\} d\right)
\end{aligned}
$$


and

$$
\begin{aligned}
& \left\langle\left\langle C_{2}\left(t_{1}-\tau_{1}^{\prime}, t_{2} ; t_{2}-\tau_{2}^{\prime}, t_{1}\right)\right\rangle\right\rangle \\
& \quad=\exp \left[-i \omega_{\mathrm{L}}\left(\tau_{1}^{\prime}-\tau_{2}^{\prime}\right)\right] \operatorname{Tr} d Z_{-}\left(\tau_{2}^{\prime}\right)\left\{d^{\dagger} Z_{0}\left(t_{2}-\tau_{2}^{\prime}-t_{1}\right)\left[d^{\dagger} Z_{+}\left(\tau_{1}^{\prime}\right)(\bar{\sigma} d)\right]\right\}
\end{aligned}
$$

Here $Z_{-}$follows from $Z_{0}$ by replacing $W_{0}$ by $W_{-}$in (3.9), with

$$
W_{-} \sigma=\lambda_{1}\left(P_{\mathrm{e}} \sigma P_{\mathrm{e}}+P_{\mathrm{g}} \sigma P_{\mathrm{g}}\right)+\lambda_{2} P_{\mathrm{e}} \sigma P_{\mathrm{g}} .
$$

After some rearrangements, we obtain from (4.3)-(4.5) the explicit result for the stochastic average of the quasi-distribution function

$$
\begin{aligned}
\left\langle\left\langle I_{2}\left(t_{1}, \omega_{1} ; t_{2}, \omega_{2}\right)\right)\right\rangle & =\frac{A^{2}}{2 \pi^{2}} \operatorname{Re} \int_{0}^{t_{2}-t_{1}} \mathrm{~d} \tau_{2}^{\prime} \exp \left[\mathrm{i}\left(\omega_{2}-\omega_{\mathrm{L}}\right) \tau_{2}^{\prime}\right] \operatorname{Tr} d^{\dagger} Z_{+}\left(\tau_{2}^{\prime}\right) \\
& \times\left\{\left[Z_{0}\left(t_{2}-\tau_{2}^{\prime}-t_{1}\right)\left(d^{\dagger} \frac{\mathrm{i}}{\omega_{1}-\omega_{\mathrm{L}}-L_{+}}(\bar{\sigma} d)+\text { Hermitian conjugate }\right)\right] d\right\} .
\end{aligned}
$$

The upper limit $t_{2}-t_{1}$ for $\tau_{2}^{\prime}$ is explained above. We wish to emphasise that (4.7) is not necessarily a good approximation for the quasi-distribution function for all values of $\omega_{1}, \omega_{2}$ and the time difference $t_{2}-t_{1}$. In particular, the contribution to the integral in (2.10) from values $\tau_{2}^{\prime}>t_{2}-t_{1}$ is omitted. On the other hand, for a time resolution obeying (4.1), we can substitute (4.7) into (2.8), and obtain a reliable result for the observable two-photon probability density $\bar{I}_{2}$, as argued above.

We notice that (4.7) depends on the time difference $t_{2}-t_{1}$, but not on the times $t_{2}$ and $t_{1}$ separately, as it should be in the steady state. Moreover, equation (4.7) is seen to contain a convolution between the Fourier-Laplace transforms of $Z_{+}$and $Z_{0}$, which reflects the fact that a spectrally resolved photon detection at time $t_{2}$ depends upon the evolution of the density matrix in the recent past.

When $t_{2}-t_{1}$ is large compared with the spontaneous lifetime of the excited state, the evolution operator $Z_{0}$ in (4.7) will have reached its asymptotic limit for all values of interest of $\tau_{2}^{\prime}$. In this case we obtain

$$
\left\langle\left\langle I_{2}\left(t_{1}, \omega_{1} ; t_{2}, \omega_{2}\right)\right\rangle\right\rangle=\left\langle\left\langle I_{1}\left(t_{1}, \omega_{1}\right)\right\rangle\right\rangle\left\langle\left\langle I_{1}\left(t_{2}, \omega_{2}\right)\right\rangle\right\rangle
$$

which, when substituted in equation $(2.8)$, gives a similar factorisation for $\left\langle\left\langle\bar{I}_{2}\right\rangle\right\rangle$.

The effect of binary collisions with foreign-gas particles can be included in the formal result (4.7) in exactly the same fashion as before (Nienhuis 1982, Arnoldus and Nienhuis 1983), giving rise to the appearance of the collisional relaxation operator $\Phi$ in the denominators in (4.7), and a similar modification in $Z_{+}$and $Z_{0}$. We shall not reproduce details of the derivation here. In the final result of the next section we shall simply indicate the additional contribution of collisions.

\section{Photon correlations between spectral lines}

The combination of equations (2.8) and (4.7) gives the spectrally resolved two-photon probability density for not too short time delays $t_{2}-t_{1}$. This result depends on the details on the function $s(\omega, \tau)$, which is determined by the spectrometer. 


\subsection{The three-line spectrum}

A special case of practical interest is the intensity correlation between the three different lines in the fluorescence spectrum of the two-state atom. Hence we consider the situation where $\Omega^{\prime}$, equation (3.22), is large compared with the widths of the lines, which are determined by the spontaneous decay rate $A$, the bandwidth $\lambda_{1}$ and the collision rate, and write

$$
\Omega^{\prime} \gg w
$$

where $w$ denotes the order of magnitude of the linewidths. The inequality (5.1) is obeyed for large values of the detuning $\Delta$ from resonance, or for large values of the Rabi frequency $\Omega$. The positions of the three lines in the spectrum are mainly determined by the eigenvalues of the Liouville operator $L_{\mathrm{d}}$, which is the dominant part of $L_{+}$in (3.20). It is convenient to introduce the dressed-atom eigenstates, which are eigenvectors of the dressed-atom Hamiltonian $H_{\mathrm{d}}$, defined in (3.8). The eigenstates are (Cohen-Tannoudji 1977)

$$
\begin{aligned}
& |1\rangle=|g\rangle \cos \frac{1}{2} \theta-|e\rangle \sin \frac{1}{2} \theta \\
& |2\rangle=|g\rangle \sin \frac{1}{2} \theta+|e\rangle \cos \frac{1}{2} \theta
\end{aligned}
$$

with

$$
\tan \theta=\Omega / \Delta \quad-\frac{1}{2} \pi \leqslant \theta<\frac{1}{2} \pi .
$$

The corresponding eigenvalues are

$$
E_{1}=\frac{1}{2} \hbar \Omega^{\prime} \quad E_{2}=-\frac{1}{2} \hbar \Omega^{\prime} .
$$

The Liouville operator $L_{\mathrm{d}}$ has the eigenvector in Liouville space $|1\rangle\langle 2|$ with eigenvalue $\Omega^{\prime}$, the eigenvector $|2\rangle\langle 1|$ with eigenvalue $-\Omega^{\prime}$, and the two eigenvectors $|2\rangle\langle 2|$ and $|1\rangle\langle 1|$ with eigenvalue 0 . The three lines in the fluorescence spectrum correspond to these three eigenvalues, and are positioned at $\omega=\omega_{\mathrm{L}}+\Omega^{\prime}$ (the three-photon line, $\mathrm{T}$ ), at $\omega=\omega_{\mathrm{L}}-\Omega^{\prime}$ (the fluorescence line, F) and at $\omega=\omega_{\mathrm{L}}$ (the Rayleigh line, R). In the secular approximation (5.1), the steady-state density matrix $\bar{\sigma}$ is to a good approximation diagonal in the dressed states, as can be shown from (3.12). The result is (Arnoldus and Nienhuis 1983)

$$
\bar{\sigma}=|1\rangle n_{1}\langle 1|+| 2\rangle n_{2}\langle 2|
$$

with dressed-state populations

$$
n_{1}=p /(p+q) \quad n_{2}=q /(p+q)
$$

with

$$
\begin{aligned}
& p=A g_{\mathrm{F}}^{2}+2 \lambda_{1} g_{\mathrm{R}}^{2}+k(\Omega, \Delta) \\
& q=A g_{\mathrm{T}}^{2}+2 \lambda_{1} g_{\mathrm{R}}^{2}+k(\Omega, \Delta) .
\end{aligned}
$$

Here we introduced a notation that is convenient in the following

$$
\begin{aligned}
& g_{\mathrm{F}}=\cos ^{2} \frac{1}{2} \theta=\left(\Omega^{\prime}+\Delta\right) / 2 \Omega^{\prime} \\
& g_{\mathrm{T}}=\sin ^{2} \frac{1}{2} \theta=\left(\Omega^{\prime}-\Delta\right) / 2 \Omega^{\prime} \\
& g_{\mathrm{R}}=\cos \frac{1}{2} \theta \sin \frac{1}{2} \theta=\Omega / 2 \Omega^{\prime}
\end{aligned}
$$

and $k(\Omega, \Delta)$ is the rate of optical collisions, defined as collisions inducing a transfer between the dressed states. 
The strengths of the three lines are easily obtained from (3.20). The contribution to each separate line arises from the action of the denominator on the corresponding eigenvector subspace. We introduce the projection operators on each of these subspaces $\mathrm{F}, \mathrm{T}$ and $\mathrm{R}$ by the definitions

$$
\begin{aligned}
& \mathscr{P}_{\mathrm{F}} \sigma=|2\rangle\langle 2|\sigma| 1\rangle\left\langle 1\left|\quad \mathscr{P}_{\mathrm{T}} \sigma=\right| 1\right\rangle\langle 1|\sigma| 2\rangle\langle 2| \\
& \mathscr{P}_{\mathrm{R}} \sigma=|2\rangle\langle 2|\sigma| 2\rangle\langle 2|+| 1\rangle\langle 1|\sigma| 1\rangle\langle 1| .
\end{aligned}
$$

Then the strengths of the lines are

$$
S_{\alpha}=A \operatorname{Tr} d^{\dagger} \mathscr{P}_{\alpha}(\tilde{\sigma} d)
$$

for $\alpha=F, T, R$. The explicit results are (Arnoldus and Nienhuis 1983)

$$
S_{\mathrm{F}}=A g_{\mathrm{F}}^{2} n_{2} \quad S_{\mathrm{T}}=A g_{\mathrm{T}}^{2} n_{1} \quad S_{\mathrm{R}}=A g_{\mathrm{R}}^{2}
$$

\subsection{Photon correlations}

The intensity correlations between the three lines can be evaluated in largely the same fashion, when we take equations (2.8) and (4.7) as our starting point. We choose the smoothing function in such a way that the three lines are still separated in $\bar{I}_{1}$. Hence we choose the spectral resolution $\Delta \omega$ so as to obey the inequalities

$$
w \ll \Delta \omega \ll \Omega^{\prime}
$$

so that the inherent time resolution can be supposed to obey the inequalities

$$
\Omega^{\prime-1} \ll \Delta \tau \ll w^{-1} .
$$

Hence the passband width of the spectrometer is large compared with the linewidth, but small compared with the frequency separation between lines. We recall moreover that the time resolution is supposed to obey the inequality (4.1), which is only consistent with (5.13) when $t_{2}-t_{1} \gg \Omega^{\prime-1}$. This is a reasonable supposition, since the spectral resolution of the lines is only meaningful on a time scale large compared with their inverse frequency distance.

The frequency positions of the lines are denoted as $\omega_{\alpha}(\alpha=\mathrm{F}, \mathrm{T}, \mathrm{R})$ and we wish to evaluate the quantities $\left\langle\left\langle I_{2}\left(t_{1}, \omega_{\alpha} ; t_{2}, \omega_{\beta}\right)\right\rangle\right\rangle$, which we shall abbreviate as $I(\alpha, \beta$; $\left.t_{2}-t_{1}\right)$ for convenience. After substituting (4.7) into the stochastic average of (2.8), we obtain an involved expression containing five integrations over $\omega_{1}^{\prime}, \omega_{2}^{\prime}, \tau_{1}, \tau_{2}$ and $\tau_{2}^{\prime}$. As a result of (5.12), the integration over $\omega_{1}^{\prime}$ can be dealt with in the same fashion as in the evaluation of (5.10) from (3.20), and the last part of (4.7) gives a term $d^{\dagger} \mathscr{P}_{\alpha}(\bar{\sigma} d)$. Since the linewidth is small compared with the width $\Delta \omega$ of the function $s$, we can replace $s\left(\omega_{1}^{\prime}, \tau_{1}\right)$ by $s\left(0, \tau_{1}\right)$. The subsequent integration over $\tau_{1}$ can be performed by noting that $Z_{0}$ gives a rapidly oscillating contribution when operating on an off-diagonal part in the dressed states, which can be entirely neglected after integration, whereas the diagonal part gives a slowly varying term (at a rate of order $w$ ), which is effectively constant over times of the order of $\Delta \tau$, due to (5.13). Hence the net result is that we can restrict $Z_{0}$ to the subspace of diagonal density matrices, and the integration over $\tau_{1}$ of $s\left(0, \tau_{1}\right)$ gives rise to the factor

$$
\zeta=\int_{0}^{\infty} \mathrm{d} \tau s(0, \tau)=\left(\int_{0}^{\infty} \mathrm{d} \tau f(\tau)\right)^{2}
$$

which has the physical significance of the transmission factor of the spectrometer for 
a monochromatic steady-state light beam with a frequency coinciding with the setting frequency. The integration over $\omega_{2}^{\prime}$ limits the range of interest of $\tau_{2}^{\prime}$ values, which must be small compared with $w^{-1}$. The same considerations apply to the integrations over $\tau_{2}^{\prime}$ and $\tau_{2}$, and the final result for the intensity correlation between the various spectral lines is

$$
I(\alpha, \beta ; t)=(\zeta A)^{2} \operatorname{Tr} d^{\dagger} \mathscr{P}_{\beta}\left\{\left[\exp (-M t) \mathscr{P}_{\mathrm{R}}\left(d^{\dagger} \mathscr{P}_{\alpha}(\bar{\sigma} d)\right)\right] d\right\}
$$

for $t \geqslant 0$. Here $M$ is a two-dimensional matrix that is defined as the restriction of $i L_{0}$, equation (3.10), to the space of diagonal density matrices. When binary collisions are accounted for, we simply replace $\mathrm{i} L_{0}$ by $\mathrm{i} L_{0}+\Phi(0)$. On the basis $|1\rangle\langle 1|| 2\rangle,\langle 2|$ the matrix $M$ takes the explicit form

$$
M=\left(\begin{array}{rr}
q & -p \\
-q & p
\end{array}\right)
$$

with $p$ and $q$ given by (5.7). (Note that the stationary density matrix (5.5) is eigenvector of $M$ with eigenvalue zero.)

\subsection{Structure of the correlations}

Equation (5.15) constitutes a considerable simplification as compared with equation (4.7) and it is only justified in the special case of well separated lines, which are detected with a spectral resolution $\Delta \omega$ that discriminates the lines in a perfect way, as indicated in (5.12).

It is instructive to compare equation (5.15) with the common intensity correlation function of resonance fluorescence with no spectral resolution (Carmichael and Walls 1976)

$$
I(t)=A^{2} \operatorname{Tr} d^{\dagger}\left[\exp \left(-\mathrm{i} L_{0} t\right)\left(d^{\dagger} \bar{\sigma} d\right)\right] d .
$$

The structure is obviously the same. The spectral resolution of the line $\alpha$ gives rise to the projection operator $\mathscr{P}_{\alpha}$, which effectively projects the dipole operator $d$ onto the transition between dressed states. The projection operator $\mathscr{P}_{\mathrm{R}}$ in $(5.15)$ selects the diagonal part of the density matrix of the atom after the emission of a photon in the line $\alpha$.

The result $(5.15)$ is derived in the case that $t>0$, which means that the photon in the line $\alpha$ is detected prior to the detection of a photon in the line $\beta$. From the definition of the probability density it is obvious that for negative times the correlation functions are determined by the symmetry relation

$$
I(\alpha, \beta ; t)=I(\beta, \alpha ;-t) .
$$

We can express the correlation function (5.15) in a more compact way by introducing the operators $\mathscr{A}_{\alpha}$ for $\alpha=\mathrm{F}, \mathrm{T}, \mathrm{R}$, by the defining relation

$$
\mathscr{A}_{\alpha} \sigma=A \mathscr{P}_{\mathrm{R}}\left[d^{\dagger} \mathscr{P}_{\alpha}(\sigma d)\right] \text {. }
$$

Furthermore, we shall assume ideal transmission, and we put the transmission factor $\zeta$ equal to unity. Then the two-photon correlation function $(5.15)$ takes the form

$$
I(\alpha, \beta ; t)=\operatorname{Tr} \mathscr{A}_{\beta} \exp (-M t) \mathscr{A}_{\alpha} \bar{\sigma}
$$

Obviously, the operator $\mathscr{A}_{\alpha}$ describes the effect on a density matrix of the atom after a photon emission in the line $\alpha$. Equation (5.20) then simply reflects successively an 
emission of a photon $\alpha$, evolution of the density matrix during a time $t$, and finally an emission of a photon $\beta$. This interpretation of equation (5.20) is confirmed by noting that the line strengths $(5.11)$ obey the equations

$$
S_{\alpha}=\operatorname{Tr} \mathscr{A}_{\alpha} \bar{\sigma}
$$

The effect of the various emissions on the atomic density matrix is illustrated by the equalities

where

$$
\begin{aligned}
& \mathscr{A}_{\mathrm{F}} \sigma=A g_{\mathrm{F}}^{2} n_{2}|1\rangle\langle 1| \\
& \mathscr{A}_{\mathrm{T}} \sigma=A g_{\mathrm{T}}^{2} n_{1}|2\rangle\langle 2| \\
& \mathscr{A}_{\mathrm{R}} \sigma=A g_{\mathrm{R}}^{2} \sigma
\end{aligned}
$$

$$
\sigma=|1\rangle n_{1}\langle 1|+| 2\rangle n_{2}\langle 2| \text {. }
$$

Hence the probability of a photon in the fluorescence line is proportional to the population of state $|2\rangle$, and this emission corresponds to a transition to state $|1\rangle$. The reverse is true for a photon in the three-photon line. An emission of a Rayleigh photon does not affect the density matrix at all.

These results are in full agreement with the simple picture of the fluorescence spectrum as resulting from transitions between dressed-atom states (Cohen-Tannoudji 1977). This is illustrated in figure 1. In fact, for free atoms in a monochromatic field Cohen-Tannoudji and Reynaud (1979) have obtained similar results by applying rate equations for a cascade in a dressed atom.

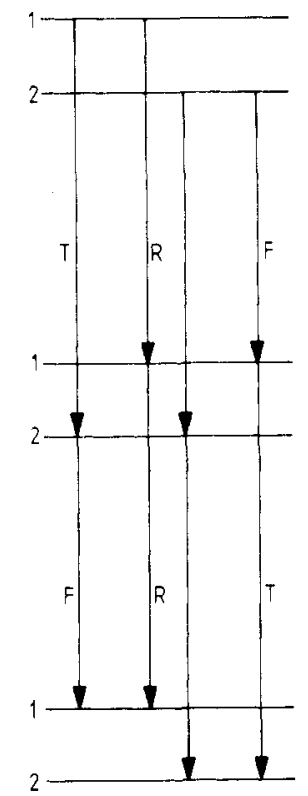

Figure 1. Illustration of the effect of emission in the various lines on the state of the dressed atom. Fluorescent emission corresponds to downward transitions in the ladder of pairs of dressed states. Emission of a photon in the three-photon line $(\mathrm{T}$ ) is seen to change the dressed state $|1\rangle$ into the dressed state $|2\rangle$, giving rise to a strongly enhanced probability for subsequent emission of a photon in the fluorescence line $(F)$. In the same way, emission of an $F$ photon corresponds to a transition from $|2\rangle$ to $|1\rangle$, giving rise to an enhancement of the probability for subsequent emission of a $T$ photon. Emission of a photon in the Rayleigh line $(R)$ leaves the state of the dressed atom unchanged. 


\section{Explicit results}

The photon correlation functions between the spectral lines of the fluorescence triplet are easily evaluated from (5.20) and (5.22), by making use of the explicit expression (5.16) for $M$. Equation (5.22c) indicates that the density matrix is not modified by emission of a Rayleigh photon, and we find that the correlation functions $I(\alpha, \beta ; t)$ simply factorise when either $\alpha$ or $\beta$ denotes the Rayleigh line (R). Hence

$$
\begin{aligned}
& I(\alpha, \mathrm{R} ; t)=S_{\alpha} S_{\mathrm{R}} \\
& I(\mathrm{R}, \beta ; t)=S_{\mathrm{R}} S_{\beta} .
\end{aligned}
$$

For positive values of $t$ the other correlation functions are found to be

$$
\begin{aligned}
& I(\mathrm{~F}, \mathrm{~F} ; t)=S_{\mathrm{F}}^{2}\{1-\exp [-(p+q) t]\} \\
& I(\mathrm{~F}, \mathrm{~T} ; t)=S_{\mathrm{F}} S_{\mathrm{T}}\{p+q \exp [-(p+q) t]\} / p \\
& I(\mathrm{~T}, \mathrm{~F} ; t)=S_{\mathrm{T}} S_{\mathrm{F}}\{q+p \exp [-(p+q) t]\} / q \\
& I(\mathrm{~T}, \mathrm{~T} ; t)=S_{\mathrm{T}}^{2}\{1-\exp [-(p+q) t]\} .
\end{aligned}
$$

The steady-state intensities $S_{\alpha}$ of the lines are given by (5.11) and (5.6). The correlation functions $I(\alpha, \beta ; t)$ for negative time values are obtained from (6.1) and (6.2) with the symmetry relation (5.1). Equations (6.1) and (6.2) generalise the results of Apanasevich and Kilin (1979) and Cohen-Tannoudji and Reynaud (1979) in that we include a finite bandwidth and collisions.

We conclude that the detection of a photon in the Rayleigh line is uncorrelated to later or previous emissions in any one of the lines. Photons from the same sideband display antibunching in time: following the detection of a photon in any one of the sidebands ( $T$ or $F$ ), a subsequent detection of a photon in the same sideband is only possible after a finite recovery time of the order of $(p+q)^{-1}$. On the other hand, photons from opposite sidebands tend to bunch: immediately following the detection of a photon in one sideband, there is an enhanced probability for detecting a photon in the other sideband. These results can be understood in a dressed-atom picture, as illustrated in figure 1 (Cohen-Tannoudji and Reynaud 1979).

These results are in line with the experiment of Aspect et al (1980). These authors measured the number of detected pairs of photons, one from the fluorescence line and one from the three-photon line, as a function of the time delay between the detections, for free atoms in the case of rather low values of $\Omega / \Delta$. In this case $p=A g_{\mathrm{F}}^{2}$ is much larger than $q=A g_{\mathrm{T}}^{2}$. When $t$ is the time delay between detection of a photon from the three-photon line and a photon from the fluorescence line, equations (6.2) show that the number of detected pairs is proportional to $1+(p / q) \exp [-(p+q) t]$ for positive delay times $t$, and proportional to $1+(q / p) \exp [(p+q) t]$ for negative delay times. Hence this number of pairs should display a dramatic increase for $t$ values just above zero. The increase for $t$ values just below zero is only very modest. In other words, the photons from the two sidebands tend to be detected in a definite time order. This is precisely what is observed by Aspect et al (1980).

At the same time, our results demonstrate that the increase gets less asymmetric around zero delay times when $\Omega / \Delta$ gets larger, and also as a result of a finite bandwidth $\lambda$, or of optical collisions, which tend to decrease the relative difference between $p$ and $q$. 
The uniform decay rate $p+q$ of all the correlation functions (6.2) is increased by collisions and by a finite bandwidth. For free atoms and monochromatic irradiation, this rate decreases with increasing $\Omega / \Delta$.

For non-monochromatic radiation we find from (5.7)

$$
p+q=A+\left(4 \lambda_{1}-2 A\right) g_{\mathrm{R}}^{2}
$$

so that $p+q$ increases for increasing values of $\Omega / \Delta$ when $\lambda_{1}>\frac{1}{2} A$. The effect of collisions is the same as the effect of a finite bandwidth. In the impact limit, the rate of optical collisions obeys the equality

$$
k=2 g_{\mathrm{R}}^{2} \gamma
$$

with $\gamma$ the low-intensity impact width. Hence the contribution of collisions to $p$ and to $q$ is accounted for by replacing $\lambda_{1}$ by $\lambda_{1}+\gamma$.

\section{Discussion}

We have introduced a general expression (2.8) and (2.9) for the two-time correlation function of spectrally resolved photons detected from a radiation field. This quantity is not a property of the field alone, but it depends also on the characteristics of the spectrometer used for the spectral resolution. This is analogous to the definition of a time-dependent spectrum (Eberly and Wódkiewicz 1977, Nienhuis 1983). The properties of the spectrometer are contained in the smoothing function $s\left(\omega^{\prime}, \tau\right)$ occurring in (2.8).

We apply this general result for the study of the photon correlations between the three lines in the spectrum of resonance fluorescence of a two-level atom. The observance of these correlation functions requires a spectral resolution $\Delta \omega$ of the spectrometer that obeys the inequalities (5.12). The general result for the correlation functions for the various combinations of two lines in the spectrum is given in equation (5.20), and explicit results are represented by (6.1) and (6.2). The general conclusion is that photons in the central Rayleigh line of the fluorescence triplet are emitted in a fully random way, with no correlation with later or previous emissions. Two photons in the same sideband display antibunching in time, whereas two photons from opposite sidebands tend to bunch. This bunching occurs in an asymmetric way, and for not too high values of $\Omega / \Delta$, detection of a photon from the three-photon line tends to precede the detection of a photon from the fluorescence line. This in line with the experimental observation by Aspect et al (1980). The behaviour of the correlation function $I(\mathrm{~T}, \mathrm{~F} ; t)$ can be characterised by the ratio

$$
I(\mathrm{~T}, \mathrm{~F} ;+0) / I(\mathrm{~T}, \mathrm{~F} ; \infty)=(p+q) / q=R_{+}
$$

and

$$
I(\mathrm{~T}, \mathrm{~F} ;-0) / I(\mathrm{~T}, \mathrm{~F} ; \infty)=(p+q) / p=R_{-}
$$

where +0 denotes the limit $t \downarrow 0$ (the $F$ photon follows the $T$ photon with very small time delay), and likewise -0 denotes the limit $t \uparrow 0$. The values of these ratios (7.1) and (7.2) as a function of $\Omega / \Delta$ are illustrated in figure 2 . A finite bandwidth tends to decrease the asymmetry in the time order, and collisions have the same effect.

The regression of the correlation function is governed by the uniform rate $p+q$, which is given by (6.3) in the absence of collisions. For low values of $\Omega / \Delta$, this rate is simply the Einstein coefficient for spontaneous emission. In the limit of large delay 


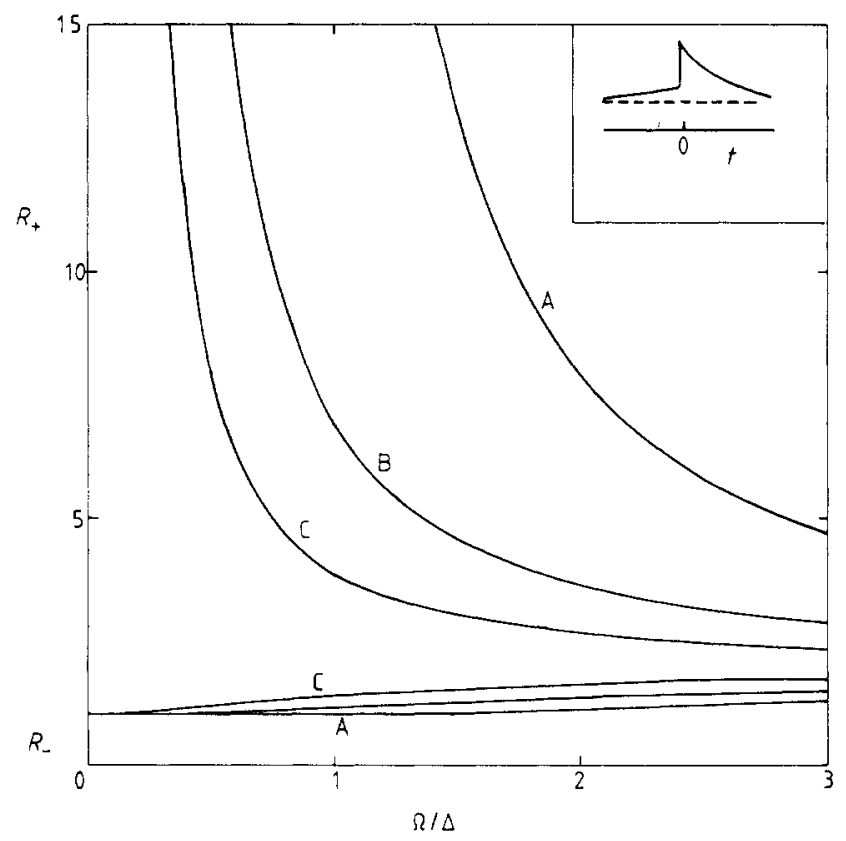

Figure 2. Plots of the enhancement factors $R_{+}$and $R_{-}$, defined in (7.1) and (7.2), characterising the values $I(\mathrm{~T}, \mathrm{~F} ; \pm 0)$. The insertion shows the qualitative behaviour of $I(\mathrm{~T}, \mathrm{~F} ; t)$. Curves $\mathrm{A}$ correspond to the case of monochromatic incident radiation in the absence of collisions $\left(\lambda_{1}+\gamma=0\right)$. Curves B correspond to the case $\lambda_{1}+\gamma=\frac{1}{2} A$, and curves $C$ to $\lambda_{1}+\gamma=\frac{3}{2} A$. The asymptotic values of $R_{+}$and $R_{-}$for $\Omega / \Delta \rightarrow \infty$ are 2 .

times $t$, the correlation functions become equal to the product of the intensities of the separate lines, as expected.

The correlations between detected photons from the two sidebands can be understood in a simple way from a dressed-atom picture, as indicated in figure 1 . The occurrence of bunching between two photons from different sidebands may seem to contradict the universal property of antibunching of two subsequently emitted photons by a two-level atom (Carmichael and Walls 1976). The solution of this apparent contradiction lies in the fact that the observance of this bunching property requires a spectral resolution better than $\Omega^{\prime}$. Therefore rapid oscillations on a time scale $\Omega^{\prime-1}$ are washed out by the limited time resolution inherent in the spectral separation of the lines. These oscillations can be observed when one foregoes any spectral resolution, and the antibunching is restored on the rapid time scale $\Omega^{\prime-1}$.

The correlation functions (5.20) for detection of two frequency-resolved photons can be directly generalised to obtain an expression for the probability density for detecting a photon in line $\alpha_{1}$ at time $t_{1}, \ldots$ and a photon in line $\alpha_{n}$ at time $t_{n}$. This $n$-fold distribution function takes the form

$$
\begin{aligned}
I_{n}\left(\alpha_{1}, t_{1} ; \ldots\right. & \left.\alpha_{n}, t_{n}\right) \\
& =\operatorname{Tr} \mathscr{A}_{\alpha_{n}} \exp \left[-M\left(t_{n}-t_{n-1}\right)\right] \ldots \mathscr{A}_{\alpha_{2}} \exp \left[-M\left(t_{2}-t_{1}\right)\right] \mathscr{A}_{\alpha_{1}} \bar{\sigma}
\end{aligned}
$$

for $t_{n}>t_{n-1} \ldots>t_{1}$. This expression is meaningful only when all the time differences $t_{i}-t_{i-1}$ are large compared with $\Omega^{\prime-1}$. This result can be applied to study the complete probability distribution of the numbers of emitted photons in the three spectral lines in a given time interval. 


\section{References}

Apanasevich P A and Kilin S Ja 1979 J. Phys. B: At. Mol. Phys. 12 L83 Arnoldus H F and Nienhuis G 1983 J. Phys. B: At. Mol. Phys. 162325

Aspect A, Roger G, Reynaud S, Dalibard J and Cohen-Tannoudji C 1980 Phys. Rev. Lett. 45617

Carlsten J L, Szöke A and Raymer M G 1977 Phys. Rev. A 151029

Carmichael H J and Walls D F 1976 J. Phys. B: At. Mol. Phys. 91199

Cohen-Tannoujdi C 1977 Frontiers in Laser Spectroscopy; Proc. 27 th Les Houches Summer School ed R Balian, S Haroche and S Liberman (Amsterdam: North-Holland)

Cohen-Tannoudji C and Reynaud S 1979 Phil. Trans. R. Soc. A 293223

Eberly J H and Wódkiewicz K 1977 J. Opt. Soc. Am. 671252

Glauber R J 1965 Quantum Optics and Electronics ed C Dewitt, A Blandin and C Cohen-Tannoudji (New York: Gordon and Breach)

Kelley P L and Kleiner W H 1964 Phys. Rev. 136 A316

Kimble H J and Mandel L 1976 Phys. Rev. A 132123

Lampard D G 1954 J. Appl. Phys. 25803

Mollow B R 1969 Phys. Rev. 1881969

Nienhuis G 1982 J. Phys. B: At. Mol. Phys. 15535

1983 J. Phys. B: At. Mol. Phys. 162677

Page C H 1952 J. Appl. Phys. 23103

Paul H 1982 Rev. Mod. Phys. 541061

Renaud B, Whitley R M and Stroud C R Jr 1977 J. Phys. B: At. Mol. Phys. 1019

Swain S 1980 Adv. At. Mol. Phys. 16159

Wódkiewicz K 1980 Phys. Lett. 77A 315 